\title{
Behavioral and Neuronal Representation of Numerosity Zero in the Crow
}

\author{
Maximilian E. Kirschhock, Helen M. Ditz, and ${ }^{(1)}$ Andreas Nieder \\ Animal Physiology Unit, Institute of Neurobiology, University of Tübingen, 72076 Tübingen, Germany
}

Different species of animals can discriminate numerosity, the countable number of objects in a set. The representations of countable numerosities have been deciphered down to the level of single neurons. However, despite its importance for human number theory, a special numerical quantity, the empty set (numerosity zero), has remained largely unexplored. We explored the behavioral and neuronal representation of the empty set in carrion crows. Crows were trained to discriminate small numerosities including the empty set. Performance data showed a numerical distance effect for the empty set in one crow, suggesting that the empty set and countable numerosities are represented along the crows' "mental number line." Single-cell recordings in the endbrain region nidopallium caudolaterale (NCL) showed a considerable proportion of NCL neurons tuned to the preferred numerosity zero. As evidenced by neuronal distance and size effects, NCL neurons integrated the empty set in the neural number line. A subsequent neuronal population analysis using a statistical classifier approach showed that the neuronal numerical representations were predictive of the crows' success in the task. These behavioral and neuronal data suggests that the conception of the empty set as a cognitive precursor of a zero-like number concept is not an exclusive property of the cerebral cortex of primates. Zero as a quantitative category cannot only be implemented in the layered neocortex of primates, but also in the anatomically distinct endbrain circuitries of birds that evolved based on convergent evolution.

Key words: corvid songbird; single-neuron recordings; nidopallium caudolaterale; numbers; empty set

Significance Statement

The conception of "nothing" as number "zero" is celebrated as one of the greatest achievements in mathematics. To explore whether precursors of zero-like concepts can be found in vertebrates with a cerebrum that anatomically differs starkly from our primate brain, we investigated this in carrion crows. We show that crows can grasp the empty set as a null numerical quantity that is mentally represented next to number one. Moreover, we show that single neurons in an associative avian cerebral region specifically respond to the empty set and show the same physiological characteristics as for countable quantities. This suggests that zero as a quantitative category can also be implemented in the anatomically distinct endbrain circuitries of birds that evolved based on convergent evolution.

\section{Introduction}

The capacity to assess quantity is not unique to humans, but deeply rooted in our animal ancestry. Comparative research, both in the wild and in the laboratory, has shown that different species of animals from diverse zoological groups can discriminate numerosity, the number of objects in a set (Butterworth et al., 2018). This includes different vertebrate species such as mammals (Brannon and Terrace, 1998), birds (Scarf et al., 2011; Vallortigara, 2017; Rugani, 2018), reptiles (Gazzola et al., 2018),

\footnotetext{
Received Jan. 15, 2021; revised Feb. 10, 2021; accepted Feb. 15, 2021.

Author contributions: M.E.K., H.M.D., and A.N. designed research; M.E.K. and H.M.D performed research;

M.E.K. and A.N. analyzed data; M.E.K. and A.N. wrote the paper; A.N. supervised the study.

This work was supported by the Deutsche Forschungsgemeinschaft Grant NI 618/2-1 (to A.N.).

The authors declare no competing financial interests.

Correspondence should be addressed to Andreas Nieder at andreas.nieder@uni-tuebingen.de.

https://doi.org/10.1523/JNEUROSCI.0090-21.2021

Copyright $\odot 2021$ the authors
}

amphibians (Stancher et al., 2015), and fishes (Agrillo and Bisazza, 2018), but also invertebrates like insects (Dacke and Srinivasan, 2008; Howard et al., 2019; Bortot et al., 2020), spiders (Cross and Jackson, 2017) and cephalopods (Yang and Chiao, 2016). In all these animals, numerical competence provides an adaptive value (Nieder, 2020a).

In addition to behavior, single-neuron correlates of countable numerosities have been described in the endbrain of humans, monkeys, and crows (Nieder et al., 2002, 2006; Nieder, 2012, 2018; Ditz and Nieder, 2015, 2016a; Viswanathan and Nieder, 2015; Kutter et al., 2018). In all three species, the number of items in a set (i.e., numerosity) is neuronally encoded by bellshaped tuning curves: neurons exhibit maximum responses to a specific preferred numerosity, and decaying activity to numerosities adjacent to the preferred one (Nieder, 2016a, 2017, 2020b). This neuronal tuning is also thought to underly numerical tuning effects of BOLD activity in human adults and children (Piazza et al., 2004; Jacob and Nieder, 2009; Kersey and Cantlon, 2017). 
While the representations of countable numerosities have been deciphered down to the level of single neurons over the past decades (Nieder, 2019), a special numerical quantity, the empty set or numerosity zero, has remained largely unexplored. The concept of zero is a late achievement in both human history and individual cognitive development (Nieder, 2016b). Conceiving of empty sets ("nothing") as a meaningful numerical category demands highlevel abstraction. Still, cognitively advanced animals possess a primitive non-symbolic notion of zero. Clear behavioral evidence of a conception of numerosity zero has so far only been reported in macaque monkeys (Merritt et al., 2009) and honeybees (Howard et al., 2018). When discriminating empty sets, both species show a numerical distance effect: they confuse numerosity 1 more often with the empty set than numerosity 2 . The empty set is therefore not only "nothing" as opposed to "something," but represented together with countable numerosities as a numerical quantity on a mental "number line" (Merritt et al., 2009; Merritt and Brannon, 2013; Nieder, 2016b).

Recently, a neuronal code for numerosity zero has only been discovered in macaque monkeys (Okuyama et al., 2015; Ramirez-Cardenas et al., 2016). Recordings in monkeys showed that single neurons in the primate cerebral cortex responded to empty sets and were tuned to preferred numerosity zero (Ramirez-Cardenas et al., 2016). However, whether the neuronal coding of numerosity zero is a special feature of the cerebral cortex or can also be found in differently designed and independently evolved endbrains is currently unknown.

Instead of a cerebral cortex, birds possess nuclear telencephalic areas as highest integration centers (Jarvis et al., 2005) that evolved independently since the last common reptilian-like ancestor of birds and mammals lived 320 million years ago (Evans, 2000). In the avian telencephalic pallium, the association area nidopallium caudolaterale (NCL) is thought to constitute a functional analog of the primate prefrontal cortex (PFC) based on anatomic and physiological parallels (Divac et al., 1985; Güntürkün, 2005; Nieder, 2017). We recently showed that neurons in the NCL respond selectively to the countable number of visual items (Ditz and Nieder, 2015, 2016a, 2020). Here, we investigated the behavioral and neuronal representation of numerosity zero (the empty set) in the carrion crow.

\section{Materials and Methods \\ Animals}

We used two male carrion crows (Corvus corone corone) of six and five years of age, Crow 1 and Crow 2, respectively. All crows were obtained from the institute's facilities. The crows were housed in social groups in indoor aviaries (Hoffmann et al., 2011). During training and experimental sessions, the crows were maintained on a controlled feeding protocol and earned food during and after the daily tests. All animal preparations and procedures complied with the National Institute of Health's Guide for Care and Use of Laboratory Animals, and were authorized by the national authority (Regierungspraesidium).

\section{Experimental apparatus}

The crows sat on a wooden perch placed inside of an operant conditioning chamber in front of a touchscreen (3 M. Microtouch, 15”, $60-\mathrm{Hz}$ refresh rate). Viewing distance to the monitor was $14 \mathrm{~cm}$. The program CORTEX (National Institute of Mental Health) presented the stimuli and stored behavioral data. An automated feeder delivered either mealworms (Tenebrio molitor larvae) or bird seed pellets on correctly completed trials. During each trial, crows were trained to keep their head still in front of the computer display. This was controlled via a reflector foil attached to the crow's head. A trial only started when the crow moved its head into the beam of an infrared light barrier and kept its head still throughout the trial, thus ensuring stable head position. Whenever the crow made premature head movements and thereby left the infrared light barrier with its head during an ongoing trial, the computer terminated the trial, and the trial was discarded.

\section{Stimuli}

We used random dot displays as numerosity stimuli. Depending on the numerosity, $0-4$ black dots $\left(0.8^{\circ}\right.$ to $1.8^{\circ}$ of visual angle $)$ were presented on a gray background $\left(12.3^{\circ}\right.$ visual angle) shown in the center of the screen. Each of these numerosities served as sample and test stimulus. The stimuli were generated using a custom-written MATLAB script. To prevent the animals from memorizing individual stimuli, multiple stimuli for each of the countable numerosities were generated anew before each session. Sample and test stimulus of the same countable numerosity were never identical within a trial. One stimulus batch contained 20 unique displays for each numerosity for each session (10 standard and 10 control displays per numerosity).

To further control for low-level visual features that may co-vary with changing numbers of dots, we showed two stimulus sets every session: the "standard" countable trial stimuli ("standard stimuli") showed dots of pseudo-random size arranged randomly (but nonoverlapping) on the background circle. The "control" countable trial stimuli ("control stimuli") showed dots with equal dot area and equal dot density across all numerosities. "Dot density" was defined as average distance between the centers of all dots on a numerosity display. "Dot area" was defined as cumulative surface area of all dots on a numerosity display, i.e., the overall black area when individual black dots were added. In addition, background luminance was varied between standard and control protocols to control for luminance differences that may occur for the empty set, and to detect their effect on neuronal responses. The mean luminance of standard stimuli at $34.4 \mathrm{~cd} / \mathrm{m}^{2}$ was higher than for control stimuli at $12.8 \mathrm{~cd} / \mathrm{m}^{2}$. To further assure that empty-set stimuli were not treated in an image-like manner, both stimulus protocols (standard and control) were presented on a circular or on a square background. Standard and control trials were randomly and unpredictably alternated.

\section{Behavioral protocol}

We trained the crows on a delayed match-to-numerosity protocol. The crow started a trial by positioning its head in front of the monitor, thus closing an infrared light barrier, and maintaining the head still throughout the trial. As soon as the crow closed the infrared light barrier, an empty green background circle was shown for $500 \mathrm{~ms}$ (baseline phase), followed by a display showing the sample numerosity. The sample stimulus disappeared after $500 \mathrm{~ms}$ and the crow had to memorize the sample for $1000 \mathrm{~ms}$ during the delay phase in which only the green background circle was visible. Head movements during sample and delay periods aborted the trial automatically. In the following test phase, the test 1 display was a match in $50 \%$ of the cases, i.e., it contained the same number of dots as the sample stimulus but differed in appearance. The crow had to respond to indicate a numerical match by moving its head out of the light barrier or by pecking at the test display. In the other $50 \%$ of the cases, the test 1 was a non-match showing either more or less dots than the sample display. Here, the crow had to refrain from responding to the test 1 stimulus (i.e., keeping its head still within the light barrier) and wait for $800 \mathrm{~ms}$. Afterwards the test 2 display appeared which always showed a match and the bird had to respond by a head movement/peck to indicate the match. Correct trials were rewarded with food via the automated feeder.

If the crow failed to respond within $800 \mathrm{~ms}$ of the relevant test period (test 1 in match trials, test 2 in non-match trials) or answered to the first test stimulus in non-match trials, the trial was counted as an error trial. As negative sensory feedback, a full-screen gray background was briefly flashed accompanied by auditory feedback, and food reward was withheld. Error trials resulted in a 5-s time-out. Prematurely aborted trials resulted in the same background flash, negative feedback tone, and 500ms time-out. Match and non-match trials, as well as different sample numerosities were presented in balanced and pseudo-random order during a session. 


\section{Surgery and recordings}

All surgeries were performed under sterile conditions while the animals were under general anesthesia. Crows were anaesthetized with a ketamine/rompun mixture (50 mg ketamine, $5 \mathrm{mg}$ xylazine/kg initially and supplemented by hourly $17 \mathrm{mg}$ ketamine, $1.7 \mathrm{mg}$ xylazine/kg, i.m.). After the surgery, the crows received analgesics [butorphanol (Morphasol), $1 \mathrm{mg} / \mathrm{kg}$, i.m.]. The head was placed in the stereotaxic holder that was customized for crows with the anterior fixation point (i.e., beak bar position) $45^{\circ}$ below the horizontal axis of the instrument. We targeted the medial part of the NCL (mNCL according to Sen et al., 2019; NCLv according to von Eugen et al., 2020), as verified histologically before (Veit and Nieder, 2013; Veit et al., 2014). This area contains numerosityselective neurons (Ditz and Nieder, 2015, 2016a, 2020; Wagener et al., 2018). The center of the craniotomy was at $5 \mathrm{~mm}$ along the anterior-posterior axis and $13 \mathrm{~mm}$ along the mediolateral axis. We recorded from the left NCL of Crow 1, and from the right NCL of Crow 2. We implanted two custom-built manual micro drives carrying four microelectrodes each (glass-coated tungsten wires, $2 \mathrm{M} \Omega$, Alpha Omega LTD). Additionally, a small connector for the head stage and a small anchor for the reflector were implanted. All implants were fixed to the bone using dental cement (Paladur, Kulzer).

At the beginning of each recording session, electrodes were advanced manually until single-cell activity was detected on at least one electrode. Neurons were sampled at random and never preselected for task involvement. Acquisition, amplification, filtering, and digitalization of neuronal data were done using a PLEXON MAP system (Plexon Inc.). Single-unit separation was performed offline (Plexon Offline Sorter, version 3.3.5)

\section{Data analysis}

All analyses were conducted in MATLAB (version R2018b, MathWorks Inc.) using custom-written software. All values in the main text and figures refer to the mean \pm standard error of the mean (SEM), unless stated otherwise. SEM was calculated as the standard deviation divided by the square root of the number of samples.

\section{Behavioral data analysis}

Behavioral performance (\% correct) was calculated by counting all correctly performed trials and divide this number by the number of all trials for each session. Percent correct performance to all stimulus combinations was calculated for each session to construct behavioral performance functions in reaction to each sample numerosity. Mean response functions were computed as the average over all individual functions per session.

\section{Neuronal selectivity and tuning analysis}

The analysis included all neurons which had a firing rate of at least $0.5 \mathrm{~Hz}$ in the relevant task window (presample onset until end of delay period) and for which at least two correct trials in each of the 20 specific conditions $(5$ sample numerosities $\times 2$ stimulus protocols $\times 2$ background shapes) were recorded.

Neuronal activity during the task was analyzed separately for the sample and delay phase. In the sample period, neuronal response rates were measured in a 500-ms window shifted by $100 \mathrm{~ms}$ from sample onset to account for the visual latency of most neurons (Veit et al., 2014). In the delay period, neuronal activity was analyzed in a 900-ms window shifted by $200 \mathrm{~ms}$ from sample offset. Since crow NCL neurons show an average visual response latency of $144 \mathrm{~ms}$ (Veit et al., 2014), the first $100 \mathrm{~ms}$ of the delay and test phase contain responses of the sample and delay period, respectively.

To determine numerosity-selectivity of the neurons, a three-factorial analysis of variance (ANOVA) was performed with factors numerosity (sample numerosities 0-4), protocol (standard and control), and background shape (circle and square). The significance threshold was set to $\alpha$ $=0.05$ and resulted in 134 (sample) and 153 (delay) numerosity-selective neurons from both crows [Table 1; a more stringent selection criterion of $\alpha=0.01$ resulted in comparable numbers of 133 (sample) and 126 (delay) numerosity-selective neurons].
Table 1. Neuronal selectivity for different task factors in a three-way ANOVA (evaluated at $\alpha=0.05$ ) on firing rates during sample and delay period

\begin{tabular}{lccc}
\hline ANOVA factor & Sample & Delay & Sample and delay \\
\hline Number & $26.7 \%(134)$ & $30.5 \%(153)$ & $12.8 \%(64)$ \\
Exclusive number & $19 \%(95)$ & $18.2 \%(91)$ & $7 \%(35)$ \\
Protocol & $10 \%(50)$ & $5.2 \%(26)$ & $1 \%(5)$ \\
Shape & $10.2 \%(51)$ & $18.6 \%(93)$ & $3.4 \%(17)$ \\
Number and protocol & $3 \%(15)$ & $1 \%(5)$ & $0 \%(0)$ \\
Number and shape & $3.8 \%(19)$ & $10.8 \%(54)$ & $0.8 \%(4)$ \\
Protocol and shape & $2 \%(10)$ & $1.2 \%(6)$ & $0.4 \%(2)$ \\
\hline
\end{tabular}

Percentages of overall recorded units ( $n=501$ neurons).

Cells with a significant main effect for factor "numerosity," but no further main effects for "protocol" or "background shape," or interaction between main effects were identified as exclusively numerosity-selective neurons. The numerosity eliciting the largest spike rate was defined as "preferred numerosity" of a given cell. Neuronal response functions of these numerosity-selective neurons were constructed by normalizing each cell's activity between its most-preferred and least-preferred numerosity. Mean neuronal response functions were computed as the average over numerosity-selective cells preferring each individual sample numerosity.

\section{Population analysis}

We trained and tested a linear multiclass support vector machine (SVM) classifier (Chang and Lin, 2011) on trial firing rates for both relevant task periods, and for different neuronal populations. For this, only cells with at least 30 trials per stimulus class, i.e., sample numerosity, were considered. The SVM classifier used one-versus-one classification to deal with five classes. Ten-fold cross-validation was performed, resulting in 27 trials for training and three trials for testing per class. Trial firing rates were $\mathrm{z}$-scored within each cross-validation repetition. The parameters for $\mathrm{z}$-scoring were derived only from the training subset of trials. We repeated the whole procedure 100 times, each time with a new set of randomly drawn trials for each cell and new cross-validation splits. To assess chance level performance of the classifier, the above procedure (including $\mathrm{z}$-scoring, cross-validation, and resamples) was also computed with shuffled trial labels. The tested SVM model offers the predicted class labels for the test subset of trials as output. Together with the true class labels for this test subset, a confusion matrix can be constructed. Values on the diagonal of this confusion matrix, divided by the absolute sum of classifications yields an accuracy measure in percent. Accuracy measures reported in the main text and figures are the mean over resamples.

\section{Error trial analysis}

We investigated an effect of error in the whole population using an SVM prediction approach. Analogue to the population analysis, a linear SVM model was trained on a subset of correct trial firing rates and then tested on a subset of either correct or error trials. For this, we considered only match error trials, since errors in non-match trials can stem from other sources than mismatching test and sample numerosity: the crows could have either left the light-barrier involuntarily during the test 1 period or prematurely respond in the anticipation of the upcoming match stimulus. Since this further narrowed down the number of error trials, we considered all neurons which had been recorded for at least one match error trial and 10 correct trials per sample numerosity. Similar to a 10 -fold cross-validation procedure, an SVM model was trained with nine correct trials per class, and then tested with either one correct or one match error trial per class. This was repeated 10 times, each time with a different split of training and test trials. Trial firing rates were normalized by $\mathrm{z}$-scoring with parameters yielded from the training subset of trials. The whole procedure was repeated 100 times, each time with a new set of randomly drawn correct and error trials for each cell. To assess chance level performance of the classifier, the above procedure (including $\mathrm{z}$ scoring, cross-validation, and resamples) was also computed with 
shuffled trial labels. Accuracy measures reported in the main text and figures are the mean over resamples.

\section{Results}

\section{Behavior}

We trained two crows on a delayed match-tosample task with numerosities from 0 to 4 (Fig. $1 A$ ). The two sets of stimuli (standard and control) controlled for low-level visual cues (Fig. $1 B)$. In the control stimulus protocol, total dot area, dot density and overall luminance were kept constant across all tested numerosities. Further, standard and control stimuli were presented on two background shapes. Presenting all stimulus sets in pseudo-random order left the crows with numerosity as only discriminating feature to successfully solve the task.

Both crows performed the task proficiently, with average performance well above $80 \%$ (Crow 1 : $84.3 \pm 0.5 \%$, 86 sessions; Crow 2: $87.3 \pm 0.4 \%, 73$ sessions). We derived behavioral performance functions for each sample numerosity (Fig. 2A,B). The values of these performance functions describe the probability the crows judged specific sample numerosities as being equal to match and all possible non-match numerosities. Both crows showed highest error rates for non-match numerosities adjacent to the respective target numerosity, with systematic improvements for more distant non-match numerosities. This effect, known as the numerical distance effect, is a clear sign that numerosities are mentally placed on an ordered quantity continuum. The numerical distance effect also holds for the empty set: Crow 1 confused the empty set more often with numerosity 1 than numerosity $2(28.1 \%$ vs $19.5 \%, p<0.001$, Wilcoxon signed-rank test, $n=86$; Fig. 2A). Crow 2, however, discriminated small numerosities more or less perfectly (Fig. $2 B$ ), so that a distance effect for the empty set, as well as numerosity one, was absent because of a ceiling effect. While performance of Crow 2 to nonmatch numerosity 1 in numerosity 0 trials was equal for standard and control trials (standard: 12.0\%; control: 12.6\%), Crow 1 erred less in the control than in the standard protocol (standard: $37.7 \%$; control: $18.7 \%)$. Because dot size for numerosity 1 was on average larger in control than in standard stimuli, dot size might have influenced empty set discrimination in Crow 1, but not in Crow 2. For both birds, the performance functions became systematically wider for increasing numerosities; an effect known as the numerical size effect, which was shown before (Ditz and Nieder, 2016b).

\section{Neuronal correlates of numerosity zero in the NCL}

While the crows performed the delayed match-to-numerosity task, we recorded the activity of 501 single-neurons (233 and 268 of crows 1 and 2, respectively) in the crows' NCL (Fig. 3A). As reported earlier, the activity of NCL neurons was modulated by countable numerosities (1-4) in both the sample and delay periods (Ditz and Nieder, 2015). More specifically, numerosity-selective neurons were tuned to individual preferred countable numerosities and showed a progressive decrease in average activity with distance from the preferred numerosity. As a new result, we report that many neurons were selectively tuned to empty set displays, numerosity zero. Figure 3 depicts the detailed activity of neurons that were tuned to numerosity zero during sample presentation, when the crows saw the empty set display (Fig. 3B,C), and the delay periods, when the crows needed to remember the empty set (Fig. 3B,D)

We quantified the number of numerosity-selective neurons separately for the sample and the delay periods using a three-way ANOVA with factors numerosity (sample numerosities 0-4), stimulus protocol (standard or control), and background shape (circle or square). For both crows together, we found that $27 \%$ $(134 / 501)$ and $31 \%(153 / 501)$ of the recorded neurons showed a main effect for numerosity during sample presentation and delay period, respectively (Table 1). Crow 2 showed $36 \%$ and $41 \%$ of significant neurons, whereas Crow 1 exhibited $16 \%$ and $19 \%$ of significant neurons during sample and delay period, respectively. On average, roughly $20 \%$ of the neurons were exclusively modulated by sample numerosity (i.e., significant main effect for numerosity without main effect for, or interaction with, any of the other factors) in at least one of the task periods of interest. These neurons were labeled numerosity-selective. Fewer neurons were found to be numerosity-selective in both task periods.

We investigated the numerosity preference of numerosityselective neurons. For each neuron, the most-preferred and leastpreferred numerosity were determined as the numerosity eliciting the highest and lowest discharge rate, respectively, in either of the relevant task periods. In agreement with previous findings in the monkey cortex (Okuyama et al., 2015; Ramirez-Cardenas et al., 2016), the most abundant class of numerosity-selective 

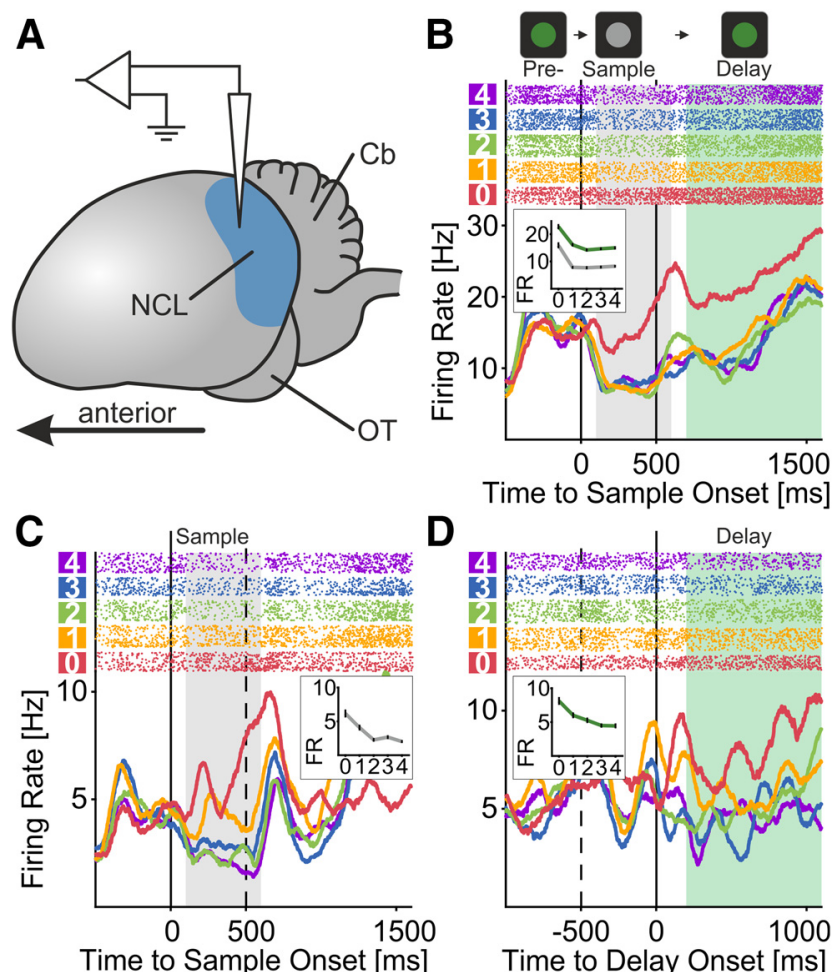

Figure 3. Empty set neurons response to numerosity. $\boldsymbol{A}$, Lateral view on schematized crow brain. Blue shaded depicts the recording site of both animals, the left NCL inside of the telencephalon. $\mathrm{Cb}$, cerebellum; $0 \mathrm{~T}$, optic tectum. $\boldsymbol{B}-\boldsymbol{D}$, Exemplary single units preferring empty set stimulus over all other countable numerosities (1-4). Top panels show dot-raster histograms (each dot representing one action potential), bottom panels show smoothed spike-density functions (smoothed with a 150-ms Gaussian kernel). The colors of dots and spike density functions correspond to the numerosity of the sample stimulus. The insets within the bottom panels show the tuning functions of the cells, averaged over time within the shaded areas (gray for sample; green for delay). Error bars show the SEM. These shaded areas also correspond to the time-windows used for all analysis of activity for the respective task period. The unit in $C$ responds maximally to empty set stimuli during the sample period, whereas neuron $(\boldsymbol{D})$ does so during the memory delay. The cell in $\boldsymbol{B}$ prefers empty set in both, sample and delay period.

neurons were those preferring the empty set. This finding was true in both the sample $(n=34,35.8 \%$ of numerosity-selective neurons; Fig. $4 B$ ) and the delay period ( $n=38,41.8 \%$; Fig. $4 D$ ). Neurons preferring each of the five numerosities were found in both crows, and in both sample and delay phases (Fig. 4B,D). Unlike in previous recordings (Ditz and Nieder, 2015), no "end effect" (i.e., an overrepresentation of the highest preferred numerosity) was observed.

We constructed population tuning curves for each preferred numerosity by averaging selective neurons' response functions normalized between the most-preferred and least-preferred numerosity (Fig. 4A,C). The average population tuning curves (which were similar for both crows individually) exhibited a clear distance effect, i.e., a gradual decrease in normalized response with increasing numerical distance from the preferred numerosity. During the sample period (Fig. 4A), this effect was evident for the empty-set neurons' population response, meaning normalized activity was significantly higher during presentation of numerosity 1 stimuli compared with numerosity 2 stimuli $(0.45$ vs $0.16, p<0.001, n=34$, Wilcoxon signed-rank test). This effect was also present in the neuron populations of each crow separately. During memory delay (Fig. $4 C$ ), no such effect could be inferred for empty-set neurons' population tuning curve $(p>0.5$, Kruskal-Wallis test). This indicates that during
A
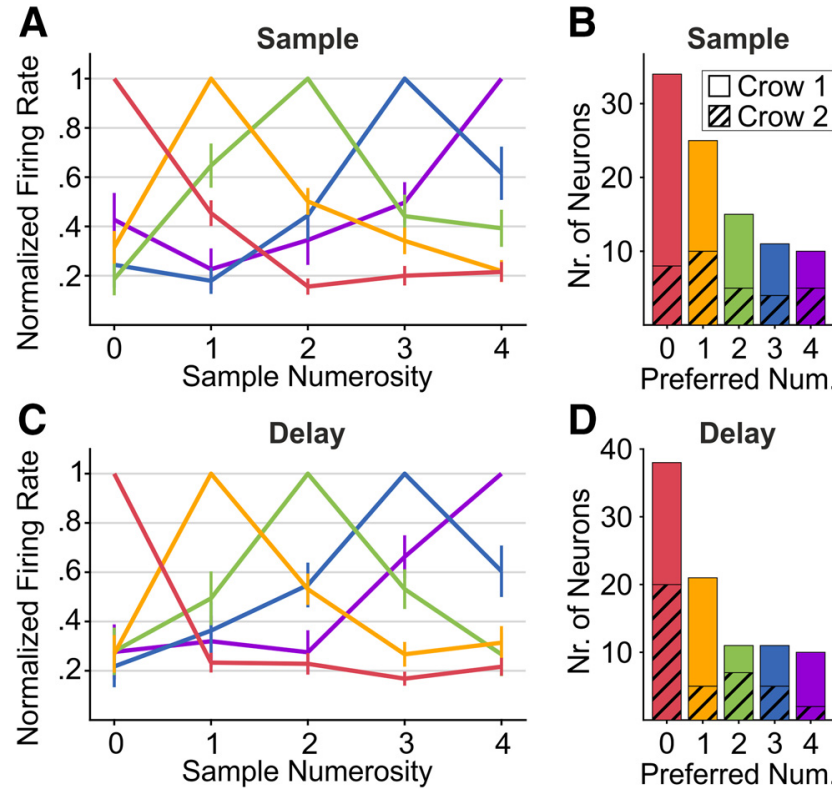

$\begin{array}{lllll}0 & 1 & 2 & 3 & 4\end{array}$

Preferred Num.

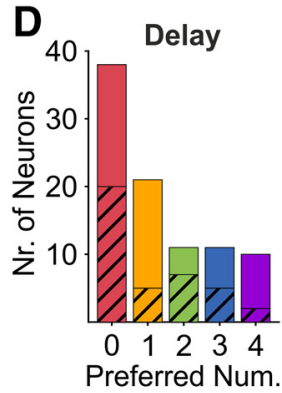

Figure 4. Population tuning curves and number preference $\boldsymbol{A}, \boldsymbol{C}$, Population tuning curves for the sample $(\boldsymbol{A})$ and delay period $(\boldsymbol{C}$ ). Curves were obtained by averaging the normalized tuning curves of all single units selectively preferring either sample numerosity (color-coded $\boldsymbol{B}, \boldsymbol{D})$. Error bars show the SEM. $\boldsymbol{B}, \boldsymbol{D}$, Incidence of neurons preferring either sample numerosity (same color-code as for the tuning curves), during either sample $(\boldsymbol{B})$ or delay $(\boldsymbol{D})$ period. Bottom parts (hatched) of stacked bars show the incidence for Crow 1, the top parts for Crow 2.

retention in working memory, empty-set neurons did differentiate between empty sets and countable numerosities, but not among the latter.

\section{Decoding empty sets from population activity}

To test whether the population of neurons, including unselective and numerosity-selective neurons, carried robust information about the continuum of numerical values, we trained and tested a statistical classifier with neuronal responses. We trained linear SVM classifiers to discriminate numerosity based on the activity of all recorded neurons with at least 30 trials per numerosity and protocol ( $n=463$; neurons from both crows pooled) in the sample and delay periods separately. The resulting confusion matrix in Figure 5 shows that the SVM classifiers performed clearly above chance level of $20 \%$ for the five numerical values (0-4). Accuracy was at $77.7 \%$ ( $\pm 3.5 \%$; mean $\pm \mathrm{SD}$ over resamples) during the sample period (Fig. $5 A$ ), and at $70.0 \%( \pm 3.9 \%)$ in the delay period (Fig. 5B). Classifier performance was also significantly above chance when equalized numbers of neurons $(n=210)$ of the two crows were tested separately, albeit with lower accuracies because of the reduced neuron count [Crow 2: 73\% (sample) and 68\% (delay); Crow 1: 44\% (sample) and 37\% (delay)]. The reduced accuracies in Crow 1 relative to Crow 2 can be explained by the lower proportion of numerosity-selective neurons found in Crow 1 .

Classifier performance exhibited characteristics seen in behavioral tuning functions: as a reflection of the numerical distance effect, most misclassifications were made in the direct numerical vicinity of the correct class, resulting in tuning functions with mild variance (Fig. $5 A, B$, top panels). As a reflection of the numerical size effect, accuracies were decreasing along the diagonal of the confusion matrices (Fig. 5A, $B$, bottom panels). Qualitatively identical results were found if the SVM classifier was trained and tested with only the numerosity-selective 
subpopulation ( $n=90$ units during sample, $n=87$ in delay). These results demonstrate that an ideal observer could determine sample numerosity, especially numerosity zero, based on the population activity of the NCL, and primarily based on the subpopulation of numerosity-selective neurons.

\section{Behavioral relevance of neuronal responses}

To test whether the neuronal responses to countable and empty set numerosities were behaviorally relevant to the crows, we performed statistical classifier analyses for activity recorded during correct and error trials. The rationale was that if neuronal activity is behaviorally meaningful, the classifier should be able to better predict the true numerical value in correct versus error trials. An SVM classifier was trained to discriminate numerosity based on the whole population's firing rates during correct trials and was subsequently used to predict the numerosity of correct or error trials. We argued that if the population code during error trials was different from correct trials, prediction accuracy should be lower in erroneous trials, thereby demonstrating behavioral relevance of said population signal. For this analysis, we only considered errors in match trials (see Materials and Methods). For correct trials, we find average prediction accuracy well above chance level (20\%, for five classes, i.e., sample numerosities) in both, sample $(52.1 \pm 8.9 \%$, mean \pm SD over resamples, $n=104$ units $)$ and delay period $(50.6 \pm 7.9 \%, n=107)$. The prediction performance exhibited characteristics shown for behavior, population tuning functions and SVM classification, namely numerical distance and size effects (Fig. 5C,D, green confusion matrices). Surprisingly, average performance dropped considerably for the prediction of numerosity in error trials, again in both sample $(33.8 \pm 9.16 \%)$ and delay $(35.3 \pm 10.9 \%)$. Numerical effects also tended to disappear (Fig. $5 C, D$, red confusion matrices). This drop in prediction accuracy was also evident for empty set trials: during sample, prediction of correct empty set trials (average accuracy $=80.4 \%$ ) was significantly higher than for error trials (vs $66.8 \%, n=100, p<0.001$, Wilcoxon signed-rank test). Same was true for the delay period $(77.8 \%$ vs $60.3 \%, n=100, p<0.001$, Wilcoxon signed-rank test). In sum, these results prompt a behavioral relevance of the NCL's population code, especially for empty set trials.

\section{Discussion}

Empty sets as part of the crow numerical continuum

Both crows robustly discriminated the empty set from other numerosities, albeit with different performance characteristics. Crow 1 exhibited a clear numerical distance effect for numerosity zero and confused the empty set with numerosity 1 more frequently than with numerosity 2 . This distance effect for the empty set was absent in Crow 2, which showed perfect discrimination performance and lack of representational variance for small numerosities; a distance effect was even absent for the discrimination of numerosity 1 versus 2 . We suspect that this perfect performance of Crow 2, which had participated in several numerosity studies before the current study (Ditz and Nieder, 2015, 2016a, 2020; Ditz et al., 2018), was a consequence of a ceiling-effect after years-long practice with dot displays. However, the behavior of Crow 1 indicates that these birds do position empty sets at the lower end of the numerical continuum.

This behavioral pattern has previously been described in only two animal species, the rhesus macaque (Merritt et al., 2009; Ramirez-Cardenas et al., 2016; Ramirez-Cardenas and Nieder, 2019) and the honeybee (Howard et al., 2018), as well as in preverbal infants (Merritt and Brannon, 2013). As our first main finding, we showed that crows can represent empty sets as a numerical null value, which is a novelty among birds. This speaks for a shared, but independently evolved non-symbolic representation of numerosity zero at the lower bound of the number line in these taxa as a conceptual precursor of the number zero. The emergence of nothing as a numerical quantity likely rests on an antecedent understanding of nothing as non-quantitative object category (Nieder, 2016b), a representation developing already in domestic chicks (Szabó et al., 2021).

\section{Corvid neurons tuned to empty sets}

In addition to the previously described single units representing countable numerosities (Ditz and Nieder, 2015, 2016a, 2020), we found a large proportion of cells selectively increasing their firing 
rate in response to empty set stimuli. Corvid neurons tuned to the empty set therefore are the second main finding of this study. Empty set neurons not only represent the subjective absence of a stimulus (Nieder et al., 2020), but the numerical category zero as part of a numerical continuum. So far, such neurons tuned to the empty set have only been reported for the primate ventral intraparietal area (VIP) and PFC (Okuyama et al., 2015; RamirezCardenas et al., 2016; Ramirez-Cardenas and Nieder, 2019). We now show that also neurons in the independently and anatomically distinctly evolved avian endbrain lacking a six-layered neocortex represent null quantity (Jarvis et al., 2005).

In macaque association cortices, neuronal responses to empty sets suggest a cortical processing hierarchy along which empty sets are steadily detached from visual properties and gradually positioned into a numerical continuum. Neurons encoding empty sets as numerical quantity during stimulus presentation (with a neuronal distance effect) are found in PFC at the apex of the processing hierarchy (Ramirez-Cardenas et al., 2016). In contrast, many VIP neurons operating upstream from PFC do not show this quantitative signature; instead, they lack a neuronal distance effect and represent the absence of items categorically (Okuyama et al., 2015; Ramirez-Cardenas et al., 2016). The graded empty sets tuning functions of crow NCL neurons during sample presentation are more reminiscent to monkey PFC neurons. This finding is consistent with the idea of NCL representing a functional analog of PFC. It also suggests that an avian brain area upstream from NCL, such as the entopallium, might contain neurons that treat nothing still as a non-quantitative category.

Differences in the tuning of empty-set neurons in NCL are also observed between the sample presentation phase and the subsequent working memory phase. Empty-set neurons showed a neuronal distance effect with gradually decreasing responses to increasing countable numerosities during the sample period, but a categorical "null-versus-countable" code lacking a graded response during the delay period. Interestingly, this change in tuning along the time course and phases of the delayed matchto-numerosity trial mirrored the findings in macaques. In macaque PFC, neurons also coded numerosity zero in a graded fashion during sample presentation (Ramirez-Cardenas et al., 2016), whereas the code changed to a categorical type during the memory delay (Ramirez-Cardenas and Nieder, 2019). We suspect that both in monkeys and in the crows, a categorical code during working memory may be advantageous in terms of coding economy and resilience against distraction.

Neuronal coding differences as a function of task periods have also been reported for other numerical tasks. In a recent crow study, NCL neurons showed a format-dependent code during the presentation of simultaneous and sequential numerosities, but a format-independent code integrating across simultaneous and sequential numerosity during the memory delay (Ditz and Nieder, 2020). The same transformation of numerical code was seen in the monkey cortex (Nieder et al., 2006). This suggests analogous transformations of numerical codes as a function of sensory and cognitive factors in both vertebrate taxa.

Behavioral relevance of numerosity information in the NCL In order to probe the information contained about numerosity in the population of NCL neurons, we used a statistical classifier approach. Based on the recorded single-cell activity, the classifier could predict both the empty set and countable numerosities with high accuracy. This points at a robust rate code for numerical quantity in the crow NCL. A numerical distance and size effect surfaced for the neuronal population analysis which mirrored not only the crows' behavioral effects, but also neuronal decoding in the primate cortex (Ramirez-Cardenas et al., 2016; Ramirez-Cardenas and Nieder, 2019). Strong support for the behavioral relevance of NCL activity for the crows' discrimination behavior was provided by an analysis of error trials. The classifier's predictions were defective when based on neuronal activity recorded during error trials. The population code seemed to become noisier and less reliable well ahead of the actual behavioral mistake. This effect of error was especially prominent for the empty set. The relevance of NCL activity for the crows' empty-set discriminations is in line with previous observations concerning the coding of behaviorally-relevant rules (Veit and Nieder, 2013; Rinnert and Nieder, 2021), memory contents (Veit et al., 2014; Rinnert et al., 2019), associations (Moll and Nieder, 2015; Veit et al., 2015), and countable numerosities (Ditz and Nieder, 2015, 2016a, 2020).

\section{Phylogenetic precursors of a concept of zero}

Our findings of a behavioral and neuronal representation of a non-symbolic precursor of a concept of zero in crows suggest two important conclusions. First, the neuronal mechanisms which facilitate quantitative understanding of the empty set must have evolved independently in at least three taxa: mammals, birds, and arthropods (insects). Second, despite the radically different anatomy of the avian endbrain, associative endbrain nuclei such as the NCL have the capacity to represent the absence of stimuli, nothing as a quantitative category. NCL neurons thus seem to achieve the same computations as the high-end neocortex of the primate brain.

\section{References}

Agrillo C, Bisazza A (2018) Understanding the origin of number sense: a review of fish studies. Philos Trans R Soc B Biol Sci 373:20160511.

Bortot M, Regolin L, Vallortigara G (2020) A sense of number in invertebrates. Biochem Biophys Res Commun. Advance online publication. Retrieved Dec 3, 2020. doi: 10.1016/j.bbrc.2020.11.039.

Brannon EM, Terrace HS (1998) Ordering of the numerosities 1 to 9 by monkeys. Science 282:746-749.

Butterworth B, Gallistel CR, Vallortigara G (2018) Introduction: the origins of numerical abilities. Philos Trans R Soc B Biol Sci 373:20160507.

Chang CC, Lin CJ (2011) LIBSVM: a library for support vector machines. ACM Trans Intell Syst Technol 2:1-27.

Cross FR, Jackson RR (2017) Representation of different exact numbers of prey by a spider-eating predator. Interface Focus 7:20160035.

Dacke M, Srinivasan M V (2008) Evidence for counting in insects. Anim Cogn 11:683-689.

Ditz HM, Nieder A (2015) Neurons selective to the number of visual items in the corvid songbird endbrain. Proc Natl Acad Sci 112:7827-7832.

Ditz HM, Nieder A (2016a) Sensory and working memory representations of small and large numerosities in the crow endbrain. J Neurosci 36:1204412052 .

Ditz HM, Nieder A (2016b) Numerosity representations in crows obey the Weber-Fechner law. Proc R Soc B Biol Sci 283:20160083.

Ditz HM, Nieder A (2020) Format-dependent and format-independent representation of sequential and simultaneous numerosity in the crow endbrain. Nat Commun 11:686.

Ditz HM, Kupferman JK, Nieder A (2018) Neurons in the hippocampus of crows lack responses to non-spatial abstract categories. Front Syst Neurosci 12:1-33.

Divac I, Mogensen J, Björklund A (1985) The prefrontal "cortex" in the pigeon. Biochemical evidence. Brain Res 332:365-368.

Evans S (2000) Evolutionary developmental biology of the cerebral cortex. In: Novartis foundation symposium 228 (BockG, CardewG, eds), pp 109113. Hoboken: Wiley.

Gazzola A, Vallortigara G, Pellitteri-Rosa D (2018) Continuous and discrete quantity discrimination in tortoises. Biol Lett 14:20180649. 
Güntürkün O (2005) The avian "prefrontal cortex" and cognition. Curr Opin Neurobiol 15:686-693.

Hoffmann A, Rüttler V, Nieder A (2011) Ontogeny of object permanence and object tracking in the carrion crow, Corvus corone. Anim Behav 82:359-367.

Howard SR, Avarguès-Weber A, Garcia JE, Greentree AD, Dyer AG (2018) Numerical ordering of zero in honey bees. Science 360:1124-1126.

Howard SR, Avarguès-Weber A, Garcia JE, Greentree AD, Dyer AG (2019) Symbolic representation of numerosity by honeybees (Apis mellifera): matching characters to small quantities. Proc R Soc B Biol Sci 286:20190238.

Jacob SN, Nieder A (2009) Tuning to non-symbolic proportions in the human frontoparietal cortex. Eur J Neurosci 30:1432-1442.

Jarvis ED, Güntürkün O, Bruce L, Csillag A, Karten H, Kuenzel W, Medina L, Paxinos G, Perkel DJ, Shimizu T, Striedter G, Wild JM, Ball GF, Dugas-Ford J, Durand SE, Hough GE, Husband S, Kubikova L, Lee DW, Mello CV, et al. (2005) Avian brains and a new understanding of vertebrate brain evolution. Nat Rev Neurosci 6:151-159.

Kersey AJ, Cantlon JF (2017) Neural tuning to numerosity relates to perceptual tuning in 3- 6-year-old children. J Neurosci 37:512-522.

Kutter EF, Bostroem J, Elger CE, Mormann F, Nieder A (2018) Single neurons in the human brain encode numbers. Neuron 100:753-761.

Merritt DJ, Brannon EM (2013) Nothing to it: precursors to a zero concept in preschoolers. Behav Processes 93:91-97.

Merritt DJ, Rugani R, Brannon EM (2009) Empty sets as part of the numerical continuum: conceptual precursors to the zero concept in rhesus monkeys. J Exp Psychol Gen 138:258-269.

Moll FW, Nieder A (2015) Cross-modal associative mnemonic signals in crow endbrain neurons. Curr Biol 25:2196-2201.

Nieder A (2012) Supramodal numerosity selectivity of neurons in primate prefrontal and posterior parietal cortices. Proc Natl Acad Sci 109:1186011865.

Nieder A (2016a) The neuronal code for number. Nat Rev Neurosci 17:366382

Nieder A (2016b) Representing something out of nothing: the dawning of zero. Trends Cogn Sci 20:830-842.

Nieder A (2017) Inside the corvid brain-probing the physiology of cognition in crows. Curr Opin Behav Sci 16:8-14.

Nieder A (2018) Evolution of cognitive and neural solutions enabling numerosity judgements: lessons from primates and corvids. Philos Trans R Soc B Biol Sci 373:20160514.

Nieder A (2019) A brain for numbers: the biology of the number instinct. Cambridge: MIT Press.

Nieder A (2020a) The adaptive value of numerical competence. Trends Ecol Evol 35:605-617.

Nieder A (2020b) Neural constraints on human number concepts. Curr Opin Neurobiol 60:28-36.

Nieder A, Freedman DJ, Miller EK (2002) Representation of the quantity of visual items in the primate prefrontal cortex. Science 297:1708-1711.

Nieder A, Diester I, Tudusciuc O (2006) Temporal and spatial enumeration processes in the primate parietal cortex. Science 313:1431-1435.

Nieder A, Wagener L, Rinnert P (2020) A neural correlate of sensory consciousness in a corvid bird. Science 369:1626-1629.
Okuyama S, Kuki T, Mushiake H (2015) Representation of the numerosity 'zero' in the parietal cortex of the monkey. Sci Rep 5:10059.

Piazza M, Izard V, Pinel P, Le Bihan D, Dehaene S (2004) Tuning curves for approximate numerosity in the human intraparietal sulcus. Neuron 44:547-555.

Ramirez-Cardenas A, Nieder A (2019) Working memory representation of empty sets in the primate parietal and prefrontal cortices. Cortex 114:102-114.

Ramirez-Cardenas A, Moskaleva M, Nieder A (2016) Neuronal representation of numerosity zero in the primate parieto-frontal number network. Curr Biol 26:1285-1294.

Rinnert P, Nieder A (2021) Neural code of motor planning and execution during goal-directed movements in crows. J Neurosci. Advance online publication. Retrieved Feb 18, 2021. doi: 10.1523/JNEUROSCI.073920.2021

Rinnert P, Kirschhock ME, Nieder A (2019) Neuronal correlates of spatial working memory in the endbrain of crows. Curr Biol 29:2616-2624.

Rugani R (2018) Towards numerical cognition's origin: insights from dayold domestic chicks. Philos Trans R Soc B Biol Sci 373:20160509.

Scarf D, Hayne H, Colombo M (2011) Pigeons on par with primates in numerical competence. Science 334:1664.

Sen S, Parishar P, Pundir AS, Reiner A, Iyengar S (2019) The expression of tyrosine hydroxylase and DARPP-32 in the house crow (Corvus splendens) brain. J Comp Neurol 527:1801-1836.

Stancher G, Rugani R, Regolin L, Vallortigara G (2015) Numerical discrimination by frogs (Bombina orientalis). Anim Cogn 18:219-229.

Szabó E, Chiandetti C, Téglás E, Versace E (2021) Young domestic chicks spontaneously represent the absence of objects. bioRxiv 427266 .

Vallortigara G (2017) An animal's sense of number. In: The nature and development of mathematics: cross disciplinary perspectives on cognition, learning and culture (Adams J, Barmby P, Mesoudi A, eds), pp 43-65. New York: Routledge.

Veit L, Nieder A (2013) Abstract rule neurons in the endbrain support intelligent behaviour in corvid songbirds. Nat Commun 4:2878.

Veit L, Hartmann K, Nieder A (2014) Neuronal correlates of visual working memory in the corvid endbrain. J Neurosci 34:7778-7786.

Veit L, Hartmann K, Nieder A (2015) Spatially tuned neurons in corvid nidopallium caudolaterale signal target position during visual search. Cereb Cortex 27:1103-1112.

Viswanathan P, Nieder A (2015) Differential impact of behavioral relevance on quantity coding in primate frontal and parietal neurons. Curr Biol 25:1259-1269.

von Eugen K, Tabrik S, Güntürkün O, Ströckens F (2020) A comparative analysis of the dopaminergic innervation of the executive caudal nidopallium in pigeon, chicken, zebra finch, and carrion crow. J Comp Neurol 528:2929-2955.

Wagener L, Loconsole M, Ditz HM, Nieder A (2018) Neurons in the endbrain of numerically naive crows spontaneously encode visual numerosity. Curr Biol 28:1090-1094

Yang TI, Chiao C-C (2016) Number sense and state-dependent valuation in cuttlefish. Proc R Soc B Biol Sci 283:20161379. 